\title{
Development of patient-derived xenograft models of prostate cancer for maintaining tumor heterogeneity
}

\author{
Changhong Shi ${ }^{1,2}$, Xue Chen ${ }^{1,2}$, Dengxu $\operatorname{Tan}^{1}$ \\ ${ }^{1}$ Division of Cancer Biology, Laboratory Animal Center, the Fourth Military Medical University, Xi'an 710032, China; ${ }^{2}$ School of Basic Medical \\ Sciences, the Chengdu Medical University, Xindu 610500, China \\ Contributions: (I) Conception and design: All authors; (II) Administrative support: None; (III) Provision of study materials or patients: None; (IV) \\ Collection and assembly of data: None; (V) Data analysis and interpretation: D Tan; (VI) Manuscript writing: All authors; (VII) Final approval of \\ manuscript: All authors. \\ Correspondence to: Changhong Shi. Laboratory Animal Center, the Fourth Military Medical University, 169 Changle West Road, Xi'an 710032 , \\ China. Email: changhong@fmmu.edu.cn.
}

\begin{abstract}
Prostate cancer (Pca) is a heterogeneous disease with multiple morphological patterns. Thus, the establishment of a patient-derived xenograft (PDX) model that retains key features of the primary tumor is of great significance. This review demonstrates the characteristics and advantages of the Pca PDX model and summarizes the main factors affecting the establishment of the model. Because this model well recapitulates the diverse heterogeneity observed in the clinic, it was extensively utilized to discover new therapeutic targets, screen drugs, and explore metastatic mechanisms. In the future, clinical phenotype and different stages of the Pca patient might be faithfully reflected by PDX model, which provides tremendous potential for understanding Pca biology and achieving individualized treatment.
\end{abstract}

Keywords: Castration-resistant prostate cancer; patient-derived xenograft (PDX); heterogeneity; individualized treatment.

Submitted Feb 19, 2019. Accepted for publication Aug 02, 2019.

doi: $10.21037 /$ tau.2019.08.31

View this article at: http://dx.doi.org/10.21037/tau.2019.08.31

\section{Introduction}

Prostate cancer (Pca) is a heterogeneous disease characterized by variation in terms of gene expression, structural genomic aberrations, epigenetic regulation, and responses to therapeutics. Although androgen deprivation therapy rapidly inhibits Pca progression, tumors often relapse as castrate-resistant prostate cancer (CRPC), which is untreatable (1). The high clinical and biological heterogeneity has hampered the development of novel therapeutics. Thus, animal models that capture the diversity and progression of Pca are urgently required and expected to provide essential references for the treatment of Pca patients (2). An alternative approach is developing Pca patient-derived xenografts (PDXs) that are initially established and subsequently passaged in immunodeficiency mice (3). This article reviews the characteristics and factors affecting Pca PDX models, focusing on its heterogeneity and implications in individualized treatment. It provides a novel tool to understand Pca progression, response to therapy, and personalized oncology.

\section{Xenograft model using Pca cell lines}

The main cell lines commonly used to establish animal models (cell-derived xenograft; CDX) of Pca are PC3, DU145, and LNCaP (Table 1) (4-10). They provide an essential foundation for the study of Pca biology. However, these tumor cells have some significant limitations for the establishment of Pca animal models. To adapt to the in vitro growth environment, these cell lines lose the threedimensional (3D) tumor structure, hence, fail to capture the inter- and intra-tumoral heterogeneity of clinical Pca, and are unable to accurately recapitulate the major 
Table 1 Cell lines for the establishment of the prostate cancer model

\begin{tabular}{llcccc}
\hline Cell line & \multicolumn{1}{c}{ Origin } & Androgen dependence & PSA & AR & References \\
\hline LNCaP & Lymph node & + & + & + & + \\
C4-2 & LNCaP-C4 variants & - & + & - & $(4,5)$ \\
PC-3 & Bone & - & - & - & $(6)$ \\
DU145 & Brain & - & - & + & $(7,8)$ \\
VCaP & Spinal cord & + & + & $(9,10)$ \\
\hline
\end{tabular}

$A R$, androgen receptor; PSA, prostate-specific antigen.

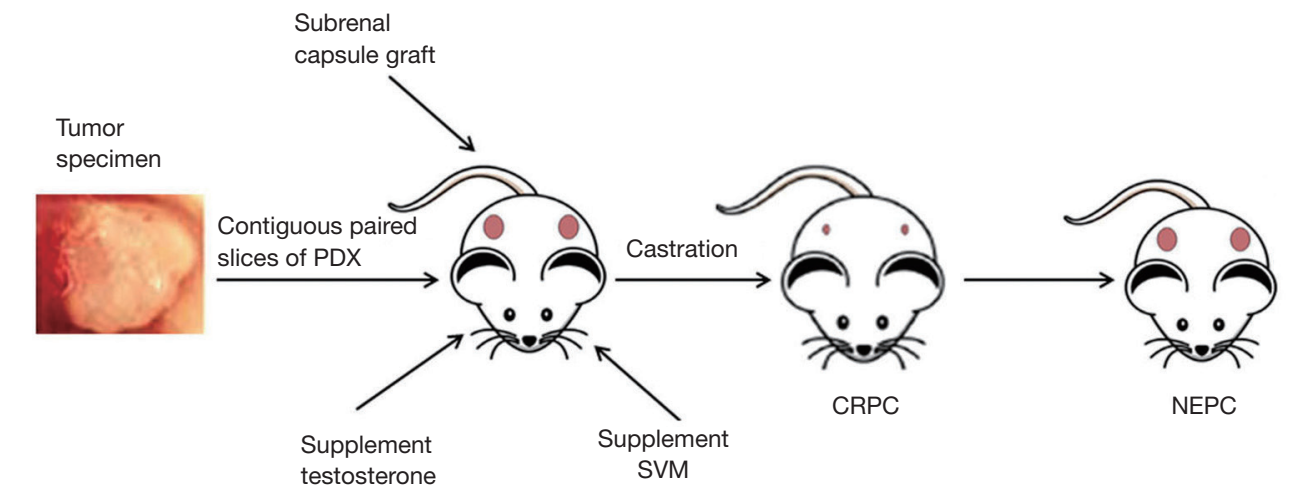

CRPC: castration-resistant prostate cancer NEPC: neuroendocrine prostate cancer

SVM: seminal vesicle mesenchyme

Figure 1 The overall procedure for the generation of prostate cancer PDX model. PDX, patient-derived xenograft.

features and diversity of human Pca. Thus, reliable data cannot be provided to support drug development, efficacy, and prognosis $(8,11,12)$. Sharpless et al. (13) also reported that tumor cell line xenografts are not able to adequately predict the efficacy of anticancer drugs, and only $5 \%$ of potential new anticancer drugs that have been tested in vivo exert significant effects and can be approved for clinical use. Currently, only 4 of the 7 classical prostate cancer cell lines reported (VCAP, LnCaP, 22Rv, MDA PCa2b) express androgen receptors (ARs) $(4,5,9,10,14-16)$, and could rarely secrete prostate-specific antigen (PSA), unlike most Pcas.

\section{Xenograft model using Pca specimens}

Currently, researchers are paying more attention to the PDX model established using Pca specimens. This type of xenograft based on intact tissue samples maintains some of the interactions with the tumor microenvironment, and more importantly, it accurately simulates the main features of Pca patients, such as hormone dependence/independence and the ability to induce castration-resistant Pca in mice by androgen ablation and other methods. The overall procedure for the generation of prostate cancer PDX model is shown as Figure 1. The development of a PDX model can promote the understanding of Pca progression, drug treatment response, and precision medicine. To date, the representative Pca PDX models include the LAPC, KUCaP, and $\mathrm{LuCaP}$ series $(8,17,18)$. An outstanding example is the LuCaP PDX series models established by Nguyen and his colleagues (19). It showed similar pathological features and growth characteristics, as well as common chromosomal changes in patients with metastatic CRPC (mCRPC). They also tested the response of this PDX model to docetaxel alone or in combination with androgen ablation treatment, and pointed out that the model can be used to study the essential features of Pca biology and evaluate the effectiveness of treatment programs. The PDX model allows human Pca tissue to grow and be passaged 
for extended periods in mice, enabling the study of tumor progression and the characteristics of various stages of the disease and testing the efficacy of novel anticancer drugs and therapeutic combinations. Although the broad spectrum of applications of PDX models in the clinic has been hampered by the long incubation period, hormonedependence, and low success rate of the graft, it more closely mimics the condition, characteristics, and treatment response of clinical patients.

\section{Factors influencing the establishment of a prostate cancer PDX model}

\section{Androgens}

Most Pcas in the clinic are androgen-dependent; however, the level of androgens in male mice is much lower than that in men. Problems such as low modeling success rate and extended incubation period are attributed to this mismatch $(20,21)$. Therefore, one of the critical factors for the establishment of a Pca PDX model is maintaining the androgen levels in mice. Supplementing mice with a specific dose of exogenous androgens promotes the growth of Pca cells and tissues (22-24) (Figure 1). Russel et al. (25) studied the effect of testosterone on the formation of prostate cancer (URCR-PR-4) from a clinical specimen in nude mice. They found that testosterone-supplemented mice (4-androsten-17ß-01-3-one) had significantly increased plasma testosterone levels and had higher tumor take rates. Despite this, just as in Risbridger's report (26), the overall success rate of Pca PDX still was extremely low (10-20\%), the time to initial growth from four up to over 12 months, and time from implantation to initial growth of secondary passage ranges from 6 to 36 weeks (19), partly due to the differences in androgen levels between human and mouse.

\section{Mouse seminal vesicle mesenchyme (SVM)}

As a unique male urogenital tumor, $\mathrm{Pca}$ is challenging to grow in mice, and it usually has a very low survival rate. Another critical factor is the lack of the stromal components and microenvironment of the donor patient's tumor to maintain or stimulate the growth of tumor tissue in the host mouse. Notably, the Leydig cells of male mammals secrete androgens and guide the differentiation and proliferation of the prostate (6). Therefore, to replicate the role of the stromal microenvironment, many laboratories have recombined human Pca tissue with the SVM, followed by co-transplantation in mice (Figure 1). Lawrence et al. (27) found that co-transplantation with SVM could support tissue growth and significantly enhanced survival and the tumor cell proliferation. These factors provide a relatively ideal microenvironment niche for Pca grafts, to obtain good tumorigenicity and maintain the critical characteristics of Pca in patients (28). In addition, clinical samples of patient tumor tissue available in laboratories are insufficient, and the stromal microenvironment provided by the SVM technology can promote the cancerous transformation of non-malignant Pca epithelial cells, thereby partially bypassing the problem of insufficient tissue used for a xenograft (29).

\section{Subrenal capsule graft}

Traditional xenograft sites for PDX models include subcutaneous, renal capsule, and orthotopic transplantation. The ideal transplant site is believed to be the orthotopic site (includes the primary site of the tumor and the metastatic site of the tumor), which provides the tumor the same anatomical microenvironment. However, for Pca, the orthotopic transplantation operation is challenging because of the limited capacity of the mouse prostate and considerable damage to host mice. Therefore, the subrenal capsule has been suggested as a suitable site for Pca xenograft (28) (Figure 1). It can significantly improve the success rate of a PDX model, which may be due to the rich vascular structure of the subrenal capsule itself, providing sufficient nutrients, hormones, and oxygen for early tumor tissue growth $(20,30)$. Compared with the other two sites, the take rate of tumor tissue by the subrenal capsule can exceed $90 \%$, and the histopathological structure, marker expression, and androgen sensitivity of the patient's tumor are faithfully reproduced $(21,31)$. Moreover, subrenal capsule xenograft has facilitated the dissection of cancer cell-microenvironment interactions in some cells with metastatic potential in primary tumors of patients, providing a valuable tool for the study of Pca metastasis mechanisms (32). In particular, combined with SVM technology, subrenal capsule xenograft can partially simulate the tumor microenvironment in orthotopic grafts and maintain the original tumor integrity (27).

\section{Tumor tissue}

High-quality Pca tissue is equally vital for establishing a PDX model. The following features of original cancer 
tissue will maximize the take rate of the xenograft (23): (I) a proportion of at least $50 \%$ of cancer; (II) high-quality undamaged tissue; and (III) the presence of proliferating cancer cells, such as those containing $\mathrm{Ki} 67^{+}$cells. Laboratory tumor samples are usually obtained from Pca biopsy, radical prostatectomy, or transurethral resection of the prostate. Samples obtained by different treatments might be in different stages of the disease, and the state of the tissue varies greatly. Pathologists need to assess the Pca tissue; curtail it precisely to reduce fat, necrosis, and other tissues; and minimize the time of ischemia after the sample is removed. Then, it should be transplanted into the mice as quickly as possible to ensure tissue vitality $(19,26,33,34)$. Simultaneously, both researchers and surgeons should be in close communication with each other to obtain continuous, clinically relevant information, including patient tumor grade, androgen dependence, PSA and AR expression, metastasis, treatment, and recurrence. These factors may not only affect the success rate of PDX model of Pca but more importantly, matching the successfully established PDX model to the corresponding patient to more accurately analyze the patient's tumor characteristics, further monitor the states of disease, and develop optimal treatment protocols. Moreover, the success rate of early Pca xenograft is extremely low because the tissue growth is often local and has low invasiveness. Therefore, most existing Pca PDX models are established using patient specimens with a high grade of advanced malignancy, which is currently an urgent problem to be solved in the primary research field of Pca $(20,35)$.

Several techniques have been applied to improve $\mathrm{PCa}$ PDXs model take rates, including the use of murine hosts with severe combined immunodeficient mice, supplementation with testosterone, subrenal implantation, and recombination of tissues with SVM. Despite these advances, the low take rate of stable PCa PDXs was reported, and the time of initial growth was 4 to over 12 months, or even longer $(19,35)$.

\section{Application}

The histopathological structure of the Pca PDX model is more similar to that of the patient's primary tumor, such as the degree of differentiation of the parental tumor, morphological features, heterogeneity, major markers, and conservation of key genes (25). It is generally believed that PDX models with few passages ( $<10$ generations) can maintain the histopathological characteristics and gene integrity of primary tumors, but some studies have also examined the molecular fidelity of $100+$ generation PDX models, which showed no significant changes in the long-term passaged mouse model, which maintains the general phenotype of PDX (19,36). The established PDX models were found to reproduce the common genome changes in clinical Pca patients (e.g., PTEN loss, RB1 loss, $A R$ amplification, and TMPRSS2-ERG fusion gene) $(19,28)$ and retained the significant markers of primary tumors in patients: AR, PSA, prostate-specific membrane antigen (PSMA), and alpha-methylacyl-CoAracemase (AMACR; also known as P504S) $(18,20,30)$. Lin et al. (35) also established some patient-derived prostate tumor xenograft models that well retained salient features of the primary tumors, including histopathology, clinical marker expression, chromosomal aberration, gene expression profiles, and molecular subtypes of prostate cancer. These results provide crucial references for studying Pca biology, identifying diagnostic markers, screening therapeutic drugs, and exploring metastatic mechanisms. The PDX model can be used as an archetype of the patient for detecting responses to various treatments and predicting treatment outcomes and prognoses to select the optimal treatment strategy for the patient.

\section{New therapeutic targets}

The metastasis capacity of the same patient at different sites has been identified by Pca PDX models. Lin et al. (37) paired metastatic and non-metastatic PDX models for gene expression analysis. The results showed that the ASAP1 gene, which may be a useful therapeutic target and/or biomarker of metastatic Pca, is involved in tumor metastasis. Terada et al. (38) used the Pca KUCaP-2 model to simulate the characteristics of clinical CRPC cases successfully and found that the prostaglandin E receptor EP4 subtype was significantly upregulated during the progression of the disease to castration resistance and could be used as a new therapeutic target for CRPC. Indeed, this laid the foundation for subsequent EP4-related studies by other researchers, and most of these studies were based on Pca cell line or cell line xenograft models for cell invasion and bone metastasis studies (39). Wick et al. (40) used the PSMAantibody-drug conjugate (PSMA-ADC) to treat cell-based xenograft Pca and PDX models. The results showed durable tumor regressions in the PDX model, demonstrating the utility of targeted PSMA therapy. In addition, researchers have used the PDX model to conduct studies on drug 
A

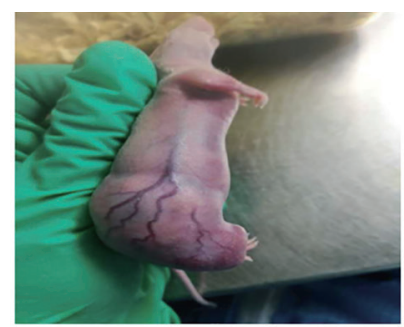

B

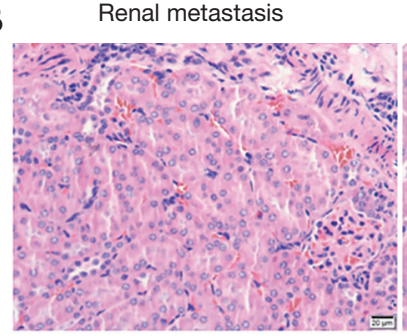

Lung metastasis

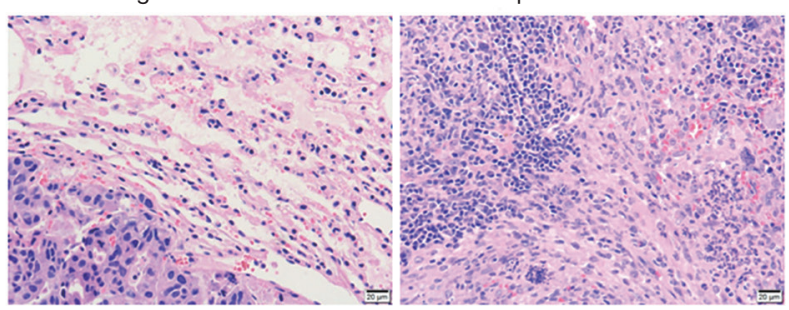

Figure 2 Multiple organ metastasis PDX models from B45354 (CRPC patient). (A) The PDX model bearing a subcutaneous transplanted tumor. (B) HE staining of metastatic tumors in organs including kidney, lung, liver, and spleen. Scale bar, $20 \mu \mathrm{m}$. PDX, patient-derived xenograft; CRPC, castrate-resistant prostate cancer.

resistance. For example, the increased expression of type III $\beta$-tubulin is closely related to docetaxel resistance, which not only results in faster postoperative recurrence but also makes patients insensitive to docetaxel and reduces the survival rate (41).

\section{Drug screening and new drug development}

Using the Pca PDX model, it is possible to screen out the most suitable drugs for patients according to the characteristics of different disease stages and to promote the development of new drugs. For example, taxanes are one of the commonly used chemotherapy drugs for Pca. Many researchers have explored taxane-based therapies, including docetaxel combined with the multivalent botanical drug (Aneustat), and docetaxel-resistant tumors treated with cabazitaxel, which both show effective anticancer activity with relatively minor side effects $(41,42)$. The poor therapeutic response of CRPC has been a major hurdle for many years. Therefore, researchers have focused on improving the therapeutic efficacy of drugs targeting CRPC. In the CRPC PDX model ( $\mathrm{LuCaP}$ 96CR), enzalutamide combined with PSMA-ADC showed potent antitumor activity and significantly increased host survival (43). Nguyen et al. (19) analysed the expression of AR in their LuCaP PDX model and found that the expression of AR transcript splice variant 7 (ARV7) was markedly upregulated in CRPC; they proposed that this model can support the development of novel AR N-terminal antagonists. Another specific type of $\mathrm{Pca}$ is neuroendocrine Pca (NEPC), which expresses synaptophysin (SYP), chromogranin A (CHGA), and chromogranin B (CHGB), while AR and PSA are usually negative or weakly expressed. Using the PDX model, Russell et al. (25) and Tung et al. (44) found that NEPCs respond well to cisplatin (or irinotecan in combination) therapy, and this approach has been used in the clinic. In addition, Beltran et al. (45) also studied the efficacy of the Aurora kinase inhibitor PHA-739358 in NEPC PDX and found that it might be beneficial to NEPC patients.

\section{Tumor metastasis research}

Establishment of metastasis models is vital to better understand the interaction of Pca cells and microenvironment. However, few Pca PDX models spontaneously metastasize from subcutaneous tumors. In one PDX model (B45354) established by our Lab from an androgen-independent Pca patient, a high metastatic potential was observed, partly due to more vascularization of the graft site (Figure $2 A$ ). Eventually, it displayed multiorgan metastases including to the kidney, lung, liver, and spleen (Figure 2B). Short tandem repeat (STR) analysis for B45354 PDX showed an intact $\mathrm{Y}$ chromosome loss. Consistent with previous 
reports, partial or complete loss of the $\mathrm{Y}$ chromosome is associated with an increased risk of cancer in men $(46,47)$. Perhaps the change in the $\mathrm{Y}$ chromosome is highly correlated with Pca metastatic potential. Even the loss of specific Y chromosome-specific genes induced the pathogenesis of Pca (48). JARID1D localized to the $\mathrm{Y}$ chromosome is a prognostic marker and suppressor of prostatic tumor invasion and metastasis (49), and Sox2 [SRY (sex determining region Y)-box 2] is an AR-repressed gene that promotes CRPC (50). Therefore, the PDX model can be used to study the genes in the $\mathrm{Y}$ chromosome involved in Pca metastasis. Orthotopic xenografts of the transplantable tumor tissue can be used for studying various aspects of metastatic prostate cancer. Pca patient's tumors were grafted in the subrenal capsule of NOD-SCID mice with testosterone-supplemented. After five serial transplantations, the tumor tissues were grafted into mouse anterior prostates. Orthotopic grafting led to metastases to multiple organs (lymph nodes, lung, liver, kidney, spleen and, notably, bone) (51). Such xenografts could therefore be useful for human metastatic prostate cancer.

\section{Patient-derived organoids (PDOs)}

Prostate cancer PDXs are costly, time consuming, and undergoing a surgical procedure. PDOs, grown in $3 \mathrm{D}$ culture with dissociated cells, is a novel pre-clinical model system in oncology that allows ex vivo propagation of tumors from individual patients (52). This $3 \mathrm{D}$ culture system for prostate tissue commonly use Matrigel for the extracellular matrix (ECM) component (53) with a liquid medium overlay to embed prostate epithelial cells. It would more closely reflect the natural behave of PCa tissue in vivo, rendering its in vitro propagation easier. When prostatic tissues are maintained in a complex matrix environment, and its utilization for prediction of therapeutic response is much more reliable.

By 3D “organoid" system, Gao et al. (54) has successfully established seven organoid lines from metastatic prostate cancer biopsies, as well as from circulating tumor cells (CTCs). These organoids faithfully recapitulated the primary tumor phenotype and their molecular profile, including phenotypic diversity of CRPC, AR-dependent adenocarcinoma, ARnegative adenocarcinoma, neuroendocrine carcinoma, and squamous differentiation. These Pca lines can be used to genetic and pharmacologic studies (55).

PDO experiments can also be performed using PDXderived tissues, allowing long-term propagated tumors to be studied in a high-throughput manner. Furthermore, PDOs can be transplanted back into mice to be grown as PDXs, allowing long-term growth in vivo. Because of their high culture take rate, it allows side-by-side comparison to evaluate the translational potential of this model system to the patient (56).

Although drug treatment of mouse prostate tumor PDO shows similar therapeutic response to that observed in vivo (57), the reliability of this system for Pca precision medicine need to be verified. One of the inherent limitations of PDO culture is the lack of blood vessels and immune cells, as well as the need to add ECM substitutes or fetal bovine. Because those substances can combine foreign factors, it may affect drug screening results. Thus, combining the advantages of PDO model and PDX will promote the process of individualized research on prostate cancer.

\section{Future perspectives}

Because PDX models preserve the heterogeneity of the primary tumor as well as the microenvironment, in the future, it might be applied to analyze a patient's clinical phenotype and simulate the phenotype of the disease at different stages. This model, therefore, has tremendous potential for individualized treatment and tumor metastasis analysis.

\section{Accurate simulation of the clinical progression of Pca}

The Pca PDX model partly simulates the transformation process and transition mechanism of the tumors from hormone-dependent to non-dependent, and from adenocarcinoma to neuroendocrine carcinoma $(18,35,58)$. There have also been studies using the PDX model to reproduce the high-risk characteristics of familial Pca: the pathological features of intraductal Pca were observed in the PDX model of familial Pca with $B R C A$ gene mutation, while these diseases usually show poor treatment effect $(26,59)$. The establishment of PDX models simulating clinical progression will allow researchers to identify and screen more effective therapeutic strategies, and patients might be able to benefit from the diversified treatment of PDX models.

\section{Platform of translation: individualized treatment}

The Pca PDX model plays a pivotal role in reducing patient 
suffering and achieving individualized treatment. Beltran et al. (60) analyzed genome sequencing information and discovered a hemizygous deletion of the FANCA gene in Pca patients. Treatment with cisplatin in the FANCA-deficient PDX model showed a cisplatin hypersensitivity response, providing biological evidence for the clinical treatment of Pca. Importantly, the characteristics and treatment response consistency of the individualized Pca PDX model and its matched patient's tumor are unmatched by other models. We also mentioned earlier that investigators and clinicians should work closely together to monitor the progress of patients dynamically, continuously obtain tumor tissue from different stages of the disease to establish paired PDX models and statistical information, and treat patients and PDX model mice in parallel. This will prove valuable in developing personalized oncology drugs, guiding the selection and adjustment of clinical treatment plans.

PDX models are lack of immune cell-tumor regulation, unable to be used in tumor immunotherapy research. The matrix components of PDX tumor tissue would be gradually replaced by matrix of mice with the number of passages increased, the environment of mice lacking the immune system also changed the microenvironment of the tumor, thus the application value of conventional PDX model was limited. This problem can be solved by humanized PDX (Hu-PDX) model. It is immune-deficient mouse engrafted with human hematopoietic stem cells (HSCs) that induce human immune system (HIS) throughout the life of the mouse (61). Usually, mice engrafted with components of HIS are routinely generated by engrafting human HSCs isolated from human fetal liver (HFL), BM, or cord blood into highly immune-deficient mouse strains (62). It could be appropriate for the screening of immune-modulating agents, such as vaccines and checkpoint blocking antibodies. This approach will allow further exploration of the role of stroma and immune system in the progression of malignancy, which will facilitate the discovery of immunotherapy targets $(63,64)$. Bartucci et al. $(65)$ reconstituted mice with a HIS from $\mathrm{PCa}$ patients along with their prostate organoids to study PCa tumor-immune cell interactions and quantify the effects of PCa therapy on immune responses to tumors. This humanized mouse would further be applied to evaluate adaptive T-cell and chimeric antigen receptor (CAR) immunotherapy.

\section{Pca metastasis analysis}

Metastasis is one of the significant factors causing a decline in the quality of life of Pca patients. Patients with metastases (including bone metastases, visceral metastases, and lymph node metastases) have a poor prognosis. At present, most models of Pca bone metastasis are established by directly injecting cell lines into the intra-femoral of mouse (66). Although the interactions between tumor cells and the bone microenvironment are known to some extent, it is not possible to study the steps of tumor cell spread and local infiltration in vivo. Unfortunately, most Pca models do not spontaneously metastasize to bone from subcutaneous tumor. Therefore, the simulation of the occurrence and development of Pca metastasis through the PDX model is one of the challenges faced in current research (32). To enhance such studies, more additional mCRPC PDX models should be generated in order to (I) exhibit both recurrent and rare molecular aberrations in AR-driven mCRPC; and (II) include the new emerging phenotype/ genotype of AR-null aggressive variant $\mathrm{Pca}$.

\section{PDX models derived from CTC}

CTC play a critical role in Pca metastasis. These tumor cells escape from the primary tumor mass and enter circulation, the most common cause of cancer related mortality. Recently, more novel methods have been developed for detection, isolation and characterization of CTCs. It has been used to generate PDX experimental models of prostate cancer (67). This approach avoids surgical sample collection and provides experimental models at various disease stages. However, the disadvantage of this method is that CTCs with metastatic potential have often undergone epithelialto-mesenchymal transition (EMT), which down regulates epithelial surface markers.

\section{Conclusions}

The prostate cancer PDX model can accurately reproduce the heterogeneity of the primary tumor and maintains good consistency with clinical results. A thorough consideration of androgen levels, SVM, transplantation sites, and transplant tissue status may improve the success rate of developing this model further. This model showed extensive application for individualized treatment, the development of new drugs, the discovery of new targets, and the exploration of Pca mechanisms in transformation studies. In the future, the ideal PDX model should faithfully reflect the distinct clinical phenotypes and different stages of the Pca patients, and offers tremendous potential for understanding Pca 
biology and achieving individualized treatment.

\section{Acknowledgments}

Funding: This work was supported by the National Natural Science Foundation Program of China (No. 31772546 and 31572340).

\section{Footnote}

Conflicts of Interest: The authors have no conflicts of interest to declare.

Ethical Statement: The authors are accountable for all aspects of the work in ensuring that questions related to the accuracy or integrity of any part of the work are appropriately investigated and resolved.

\section{References}

1. Kirby M, Hirst C, Crawford ED. Characterising the castration-resistant prostate cancer population: a systematic review. Int J Clin Pract 2011;65:1180-92.

2. Lange T, Oh-Hohenhorst SJ, Joosse SA, et al. Development and Characterization of a Spontaneously Metastatic Patient-Derived Xenograft Model of Human Prostate Cancer. Sci Rep 2018;8:17535.

3. Zhang W, van Weerden WM, de Ridder CMA, et al. Ex vivo treatment of prostate tumor tissue recapitulates in vivo therapy response. Prostate 2019;79:390-402.

4. Lim DJ, Liu XL, Sutkowski DM, et al. Growth of an androgen-sensitive human prostate cancer cell line, LNCaP, in nude mice. Prostate 1993;22:109-18.

5. Castanares MA, Copeland BT, Chowdhury WH, et al. Characterization of a novel metastatic prostate cancer cell line of LNCaP origin. Prostate 2016;76:215-25.

6. Govindaraj V, Arya SV, Rao AJ. Differential action of glycoprotein hormones: significance in cancer progression. Horm Cancer 2014;5:1-10.

7. Thomas C, Wafa LA, Lamoureux F, et al. Carbidopa enhances antitumoral activity of bicalutamide on the androgen receptor-axis in castration-resistant prostate tumors. Prostate 2012;72:875-85.

8. Toivanen R, Taylor RA, Pook DW, et al. Breaking through a roadblock in prostate cancer research: an update on human model systems. J Steroid Biochem Mol Biol 2012;131:122-31.

9. Syvälä H, Pennanen P, Bläuer M, et al. Additive inhibitory effects of simvastatin and enzalutamide on androgensensitive $\mathrm{LNCaP}$ and $\mathrm{VCaP}$ prostate cancer cells. Biochem Biophys Res Commun 2016;481:46-50.

10. Korenchuk S, Lehr JE, Mclean L, et al. VCaP, a cellbased model system of human prostate cancer. In Vivo 2001;15:163-8.

11. van Weerden WM, Bangma C, de Wit R. Human xenograft models as useful tools to assess the potential of novel therapeutics in prostate cancer. Br J Cancer 2009;100:13-8.

12. Shen MM, Abateshen C. Molecular genetics of prostate cancer: new prospects for old challenges. Genes Dev 2010;24:1967-2000.

13. Sharpless NE, Depinho RA. The mighty mouse: genetically engineered mouse models in cancer drug development. Nat Rev Drug Discov 2006;5:741-54.

14. Sramkoski RM, Pretlow TG 2nd, Giaconia JM, et al. A new human prostate carcinoma cell line, 22Rv1. In Vitro Cell Dev Biol Anim 1999;35:403-9.

15. Navone NM, Logothetis CJ, von Eschenbach AC, et al. Model systems of prostate cancer: uses and limitations. Cancer Metastasis Rev 1998;17:361-71.

16. Hara T, Nakamura K, Araki H, et al. Enhanced androgen receptor signaling correlates with the androgen-refractory growth in a newly established MDA PCa 2b-hr human prostate cancer cell subline. Cancer Res 2003;63:5622-8.

17. Patrawala L, Calhoun-Davis T, Schneider-Broussard $\mathrm{R}$, et al. Hierarchical organization of prostate cancer cells in xenograft tumors: the CD44+alpha2beta1+ cell population is enriched in tumor-initiating cells. Cancer Res 2007;67:6796-805.

18. Yoshikawa T, Kobori G, Goto T, et al. An original patient derived xenograft of prostate cancer with cyst formation. Prostate 2016;76:994-1003.

19. Nguyen HM, Vessella RL, Morrissey C, et al. LuCaP Prostate Cancer Patient-Derived Xenografts Reflect the Molecular Heterogeneity of Advanced Disease an--d Serve as Models for Evaluating Cancer Therapeutics. Prostate 2017;77:654-71.

20. Zhao H, Nolley R, Chen Z, et al. Tissue slice grafts: an in vivo model of human prostate androgen signaling. Am J Pathol 2010;177:229-39.

21. Davies AH, Wang Y, Zoubeidi A. Patient-derived xenografts: A platform for accelerating translational research in prostate cancer. Mol Cell Endocrinol 2018;462:17-24.

22. Song W, Soni V, Soni S, et al. Testosterone inhibits the growth of prostate cancer xenografts in nude mice. BMC 
Cancer 2017;17:635.

23. Lawrence MG, Pook DW, Wang H, et al. Establishment of primary patient-derived xenografts of palliative TURP specimens to study castrate-resistant prostate cancer. Prostate 2015;75:1475-83.

24. Michiel Sedelaar JP, Dalrymple SS, et al. Of mice and men--warning: intact versus castrated adult male mice as xenograft hosts are equivalent to hypogonadal versus abiraterone treated aging human males, respectively. Prostate 2013;73:1316-25.

25. Russell PJ, Russell P, Rudduck C, et al. Establishing Prostate Cancer Patient Derived Xenografts: Lessons Learned From Older Studies. Prostate 2015;75:628-36.

26. Risbridger GP, Taylor RA. Patient-Derived Prostate Cancer: from Basic Science to the Clinic. Horm Cancer 2016;7:236-40.

27. Lawrence MG, Taylor RA, Toivanen R, et al. A preclinical xenograft model of prostate cancer using human tumors. Nat Protoc 2013;8:836-48.

28. McLean DT, Strand DW, Ricke WA. Prostate cancer xenografts and hormone induced prostate carcinogenesis. Differentiation 2017;97:23-32.

29. Olumi AF, Grossfeld GD, Hayward SW, et al. Carcinomaassociated Fibroblasts Direct Tumor Progression of Initiated Human Prostatic Epithelium. Cancer Res 1999;59:5002-11.

30. Wang Y, Wang JX, Xue H, et al. Subrenal capsule grafting technology in human cancer modeling and translational cancer research. Differentiation 2016;91:15-9.

31. Wang Y, Revelo MP, Sudilovsky D, et al. Development and characterization of efficient xenograft models for benign and malignant human prostate tissue. Prostate 2005;64:149-59.

32. Lin D, Bayani J, Wang Y, et al. Development of metastatic and non-metastatic tumor lines from a patient's prostate cancer specimen-identification of a small subpopulation with metastatic potential in the primary tumor. Prostate 2010;70:1636-44.

33. Kumar A, Coleman I, Morrissey C, et al. Substantial interindividual and limited intra-individual genomic diversity among tumors from men with metastatic prostate cancer. Nat Med 2016;22:369-78.

34. Presnell SC, Werdin ES, Maygarden S, et al. Establishment of short-term primary human prostate xenografts for the study of prostate biology and cancer. Am J Pathol 2001;159:855-60.

35. Lin D, Wyatt AW, Xue H, et al. High fidelity patient-derived xenografts for accelerating prostate cancer discovery and drug development. Cancer Res 2014;74:1272-83.

36. Izumchenko E, Meir J, Bedi A, et al. Patient-derived xenografts as tools in pharmaceutical development. Clin Pharmacol Ther 2016;99:612-21.

37. Lin D, Watahiki A, Bayani J, et al. ASAP1, a gene at 8q24, is associated with prostate cancer metastasis. Cancer Res 2008;68:4352-9.

38. Terada N, Shimizu Y, Kamba T, et al. Identification of EP4 as a potential target for the treatment of castrationresistant prostate cancer using a novel xenograft model. Cancer Res 2010;70:1606-15.

39. Xu S, Zhang Z, Ogawa O, et al. An EP4 antagonist ONO-AE3-208 suppresses cell invasion, migration, and metastasis of prostate cancer. Cell Biochem Biophys 2014;70:521-7.

40. Wick M, Quinn M, Mangold A, et al. Establishment and characterization of a hormone dependent, PSA/PSMA positive prostate PDX model. Eur J Cancer 2016;69:S113.

41. de Morrée E, van Soest R, Aghai A, et al. Understanding taxanes in prostate cancer; importance of intratumoral drug accumulation. Prostate 2016;76:927-36.

42. Qu S, Wang K, Xue H, et al. Enhanced anticancer activity of a combination of docetaxel and Aneustat (OMN54) in a patient-derived, advanced prostate cancer tissue xenograft model. Mol Oncol 2014;8:311-22.

43. DiPippo VA, Nguyen HM, Brown LG, et al. Addition of PSMA ADC to enzalutamide therapy significantly improves survival in in vivo model of castration resistant prostate cancer. Prostate 2016;76:325-34.

44. Tung WL, Wang Y, Gout PW, et al. Use of irinotecan for treatment of small cell carcinoma of the prostate. Prostate 2011;71:675-81.

45. Beltran H, Rickman DS, Park K, et al. Molecular characterization of neuroendocrine prostate cancer and identification of new drug targets. Cancer Discov 2011;1:487-95.

46. Noveski P, Madjunkova S, Sukarova Stefanovska E, et al. Loss of Y Chromosome in Peripheral Blood of Colorectal and Prostate Cancer Patients. PLoS One 2016;11:e0146264.

47. Patel R, Khalifa AO, Isali I, et al. Prostate cancer susceptibility and growth linked to $\mathrm{Y}$ chromosome genes. Front Biosci (Elite Ed) 2018;10:423-36.

48. Perinchery G, Sasaki M, Angan A, et al. Deletion of Y-chromosome specific genes in human prostate cancer. J Urol 2000;163:1339-42.

49. Li N, Dhar SS, Chen TY, et al. JARID1D is a suppressor 
and prognostic marker of prostate cancer invasion and metastasis. Cancer Res 2016;76:831-43.

50. Kregel S, Kiriluk KJ, Rosen AM, et al. Sox2 Is an Androgen Receptor-Repressed Gene That Promotes Castration-Resistant Prostate Cancer. Plos One 2013;8:e53701.

51. Wang Y, Xue H, Cutz JC, et al. An orthotopic metastatic prostate cancer model in SCID mice via grafting of a transplantable human prostate tumor line. Lab Invest 2005;85:1392-404.

52. Weeber F, Ooft SN, Dijkstra KK, et al. Tumor Organoids as a Pre-clinical Cancer Model for Drug Discovery. Cell Chem Biol 2017;24:1092-100.

53. Kleinman HK, Martin GR. Matrigel: Basement membrane matrix with biological activity. Semin Cancer Biol 2005;15:378-86.

54. Gao D, Vela I, Sboner A, et al. Organoid cultures derived from patients with advanced prostate cancer. Cell 2014;159:176-87.

55. Puca L, Bareja R, Prandi D, et al. Patient derived organoids to model rare prostate cancer phenotypes. Nat Commun 2018;9:2404.

56. Risbridger GP, Toivanen R, Taylor RA. Preclinical Models of Prostate Cancer: Patient-Derived Xenografts, Organoids, and Other Explant Models. Cold Spring Harb Perspect Med 2018. doi: 10.1101/cshperspect.a030536.

57. Chua CW, Shibata M, Lei M, et al. Single luminal epithelial progenitors can generate prostate organoids in culture. Nat Cell Biol 2014;16:951-61.

58. Beltran H, Prandi D, Mosquera JM, et al. Divergent clonal evolution of castration-resistant neuroendocrine prostate cancer. Nat Med 2016;22:298-305.

Cite this article as: Shi C, Chen X, Tan D. Development of patient-derived xenograft models of prostate cancer for maintaining tumor heterogeneity. Transl Androl Urol 2019;8(5):519-528. doi: 10.21037/tau.2019.08.31
59. Risbridger GP, Taylor RA, Clouston D, et al. Patientderived xenografts reveal that intraductal carcinoma of the prostate is a prominent pathology in BRCA2 mutation carriers with prostate cancer and correlates with poor prognosis. Eur Urol 2015;67:496-503.

60. Beltran H, Eng K, Mosquera JM, et al. Whole-Exome Sequencing of Metastatic Cancer and Biomarkers of Treatment Response. JAMA Oncol 2015;1:466-74.

61. Drake AC, Chen Q, Chen J. Engineering humanized mice for improved hematopoietic reconstitution. Cell Mol Immunol 2012;9:215-24.

62. Chen DS, Mellman I. Oncology meets immunology: the cancer-immunity cycle. Immunity 2013;39:1-10.

63. Cassidy JW, Caldas C, Bruna A. Maintaining Tumor Heterogeneity in Patient-Derived Tumor Xenografts. Cancer Res 2015;75:2963-8.

64. Rea D, Vecchio VD, Palma G, et al. Mouse Models in Prostate Cancer Translational Research: From Xenograft to PDX. Biomed Res Int 2016;2016:9750795.

65. Bartucci M, Ferrari AC, Kim IY, et al. Personalized Medicine Approaches in Prostate Cancer Employing Patient Derived 3D Organoids and Humanized Mice. Front Cell Dev Biol 2016;4:64.

66. Raheem O, Kulidjian AA, Wu C, et al. A novel patientderived intra-femoral xenograft model of bone metastatic prostate cancer that recapitulates mixed osteolytic and osteoblastic lesions. J Transl Med 2011;9:185.

67. Williams ES, Rodriguez-Bravo V, Chippada-Venkata U, et al. Generation of Prostate Cancer Patient Derived Xenograft Models from Circulating Tumor Cells. J Vis $\operatorname{Exp} 2015 ;(105): 53182$. 